\title{
Use of a bis-1,2,3-triazole gelator for the preparation of supramolecular metallogels and stabilization of gold nanoparticles $\uparrow$
}

\author{
Markus Tautz, ${ }^{a}$ César Saldías, ${ }^{b}$ Antonio Diego Lozano-Gorrín ${ }^{c, d}$ and David Díaz Díaz ${ }^{*, e}$ \\ Received (in XXX, XXX) Xth XXXXXXXXX 20XX, Accepted Xth XXXXXXXXX 20XX \\ DOI: $10.1039 / b 000000 x$
}

In this work we have applied a double isosteric substitution of the amide groups presented in the gelator $N, N^{\prime}$-((1S,2S)-cyclohexane-1,2-diyl)didodecanamide $\left(\mathbf{C}_{\mathbf{1 2}}\right.$-Cyc) by 1,2,3-triazole rings affording the corresponding isosteric gelator (click- $\mathbf{C}_{\mathbf{1 2}}-\mathbf{C y c}$ ). This approach enabled the preparation of different metallogels and fine-tuning their properties. In particular, $\mathbf{C}_{\mathbf{1 2}}$-Cyc was found to form stable and

10 reversible metallogels with $\mathrm{Pd}^{\mathrm{II}}$ and $\mathrm{Au}^{\mathrm{III}}$ in DMSO and DMF, whereas click-C $\mathbf{C}_{\mathbf{1 2}}-\mathbf{C y c}$ formed metallogels in these solvents only in the presence of $\mathrm{Au}^{\mathrm{III}}$. Comparing the gels made of $\mathbf{C}_{\mathbf{1 2}}-\mathbf{C y c} v s$ click-C $\mathbf{C}_{\mathbf{1 2}}-\mathbf{C y c}$ in the presence of $\mathrm{HAuCl}_{4} \cdot 3 \mathrm{H}_{2} \mathrm{O}$, the former showed higher $T_{\text {gel }}$ values and 5-8 times slower gelation kinetics. Furthermore, the incorporation of $\mathrm{Et}_{3} \mathrm{~N}$ as reducing agent in the formulations facilitated the in situ formation of non-aggregated gold nanoparticles embedded in the viscoelastic networks made of either 15 pure isosteres or with an equimolar mixture of click-C $\mathbf{C}_{\mathbf{1 2}}-\mathbf{C y c}$ and $\mathbf{C}_{\mathbf{1 2}}-\mathbf{C y c}$. So-formed NPs showed similar size distribution regardless the gold concentration and the nature of the gelator system.

\section{Introduction}

Gels have received increasing attention during the last decades ${ }^{1,2,3}$ due to their hierarchical molecular architectures and potential 20 applications in various fields ${ }^{4,5}$ including the preparation of sensors, ${ }^{6}$ liquid crystals, ${ }^{7}$ conductive scaffolds,,${ }^{8,9}$ templates for cell growth ${ }^{10}$ and inorganic structures, ${ }^{11}$ catalysis, ${ }^{12,13}$ cosmetics $^{14}$ and food industries. ${ }^{15}$ Chemical gels are formed by covalent bonds, ${ }^{16}$ while physical (or supramolecular) gels ${ }^{17,18,19}$ are usually 25 formed by self-assembly of low-molecular-weight (LMW) molecules into 1D nanofibers that undergo further entanglement affording a $3 \mathrm{D}$ scaffold. The immobilization of solvent molecules (major component) into the interstices of the 3D network via capillary forces and surface tension can increase the viscosity of 30 the medium by factors up to $10^{10}$ and confers the solid-like appearance to the gels. Due to the non-covalent nature of the interactions (e.g. hydrogen-bonding, $\pi$-stacking, van der Waals), most physical organic gels display reversible gel-to-sol transitions that can be triggered by a variety of external stimuli 35 (e.g. temperature, $\mathrm{pH}$, light, ionic strength). ${ }^{20,21,22}$ Another important subgroup of gels are metallogels, where the main driving force for their formation is the metal-ligand coordination. Thus, the metallogels differ mainly in the metal ion used, which directly influences their functions, redox, optic, magnetic and

\footnotetext{
anstitut für Organische Chemie, Universität Regensburg, Universitätsstr. 31, 93053 Regensburg, Germany.

E-mail: David.Diaz@chemie.uni-regensburg.de

${ }^{b}$ Departamento de Química Física, Facultad de Química, Pontificia Universidad Católica de Chile, Casilla 302, Correo 22, Santiago, Chile

c Departamento de Química, Universidad de La Laguna, 38206 La Laguna, Tenerife, Spain.

' Instituto Universitario de Materiales y Nanotecnología (IMN), Universidad de La Laguna, 38200 La Laguna, Tenerife, Spain

e Instituto de Productos Naturales y Agrobiología del CSIC, Avda. Astrofísico Francisco Sánchez 3, 38206 La Laguna, Tenerife, Spain E-mail:d.diaz.diaz@ipna.csic.es

$\dagger$ Electronic Supplementary Information (ESI) available: Spectra (FT-IR, $\mathrm{XRD}$ ), tabular data, additional plots and electron microscopy images. See DOI: $10.1039 / \mathrm{b} 000000 \mathrm{x} /$
}

40 electronic properties. ${ }^{23,24,25,26,27}$

In terms of strategies for fine-tuning the properties of physical gels, our group reported a few years ago for the first time the isosteric replacement ${ }^{28}$ of amides by 1,2,3-triazole groups in LMW gelators. ${ }^{29}$ In general, isosteres possess practically equal ${ }_{45}$ molecular shape and volume, as well as similar electronic distribution and physical properties. ${ }^{30}$ As a practical concept, isosteric substitution has a long and successful history in medicinal chemistry for drug development, ${ }^{31}$ but it is still in its infancy in materials synthesis. In our previous work, we replaced ${ }_{50}$ an amide group by a 1,2,3-triazole in a glutamic acid-derived LMW gelator, where both the amide and the carboxylic acid groups played a key role on the self-assembly process via hydrogen bonds. ${ }^{29,32}$ Very recently, we reported the study of a more challenged scenario where 1,2,3-triazole rings replaces all 55 the hydrogen bond centers in a LMW gelator without sacrificing its gelation capacity. ${ }^{33}$ In particular, we carried out a double isosteric replacement of both amide groups in a known LMW gelator $\quad \mathbf{C}_{\mathbf{1 2}}$-Cyc $\quad$ (i.e. $\quad N, N^{\prime}-((1 S, 2 S)$-cyclohexane-1,2diyl)didodecanamide $)^{34,35,36,37,38}$ by $1,2,3$-triazole rings affording ${ }_{60}$ the corresponding isostere click- $\mathbf{C}_{\mathbf{1 2}}-\mathbf{C y c}$ (Figure 1). It should be emphasized that the preservation of the gelation ability upon dual isosteric substitution is not evident because the directional amide groups constitute in this case the source of hydrogen bonds to build the 3D self-assembled network. Satisfactorily, the results of ${ }_{65}$ the comparison of both gelators in terms of gelation ability and gel properties demonstrated that 1,2,3-triazoles can take over all the functions derived from the amides groups offering a versatile strategy for fine-tuning the properties of the corresponding physical gels. 


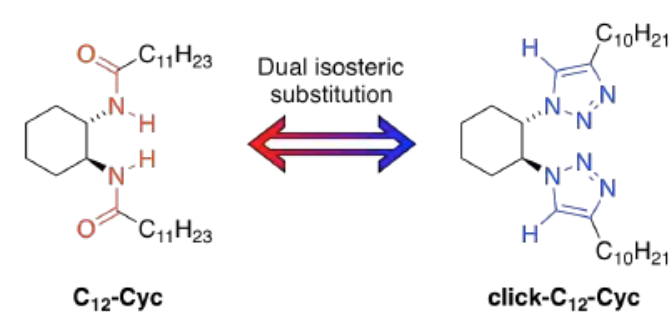

Fig. 1 Isosteric LMW gelators $\mathbf{C}_{\mathbf{1 2}}-\mathbf{C y c}$ and click- $\mathbf{C}_{\mathbf{1 2}}$-Cyc used in this work for the preparation of metallogels.

Herein, we have extended our study to the formation of 5 metallogels and in situ preparation of gold nanoparticles embedded in the gel-phase networks formed by $\mathbf{C}_{\mathbf{1 2}}-\mathbf{C y c}$ and/or click- $\mathrm{C}_{12}$-Cyc.

\section{Experimental}

\section{Materials}

10 Unless otherwise indicated, all chemicals, reagents and solvents were of analytical grade, purchased from commercial sources (Sigma-Aldrich, TCI Europe, Alfa Aesar) and used as received without further purification. Double-distilled water was purified additionally using a Millipore water-purifying system (Merck)

15 prior usage. Isosteric gelators $N, N^{\prime}-((1 S, 2 S)$-cyclohexane-1,2diyl)didodecanamide $\left(\mathbf{C}_{\mathbf{1 2}}\right.$-Cyc) and $(1 S, 2 S)$-1,2-bis(4-decyl-1H1,2,3-triazol-1-yl)cyclohexane (click- $\mathbf{C}_{\mathbf{1 2}}$-Cyc) were prepared and characterized as we have previously described. ${ }^{33}$

\section{Characterization of metallogels}

${ }_{20}$ Critical gelation concentration (CGC) values (defined as the minimum concentrations of compound where gelation is observed) were estimated by continuously adding aliquots of solvent $(0.02-0.1 \mu \mathrm{L})$ into vials containing the desired mixture of [gelator + metal salt] and performing a typical heating-cooling 25 protocol for gel-formation until no gelation was observed. The starting point for CGC determinations was $120 \mathrm{~g} \mathrm{~L}^{-1}$ in most of the cases. No further experiments were made if gelation was not achieved at $200 \mathrm{~g} \mathrm{~L}^{-1}$. Non-gel containing vials (i.e. clear solution after 7 days) were frequently left at RT and visually monitored 30 for possible gelation/crystallization over time.

Thermal gel-to-sol transition temperature $\left(T_{\text {gel }}\right)$ values were determined using a custom made set-up where the sealed vial containing $100 \mu \mathrm{L}$ of the metallogel was placed into a mold of an alumina block and heated up at $1{ }^{\circ} \mathrm{C} / 5 \mathrm{~min}$ using an electric 35 heating plate equipped with a temperature control couple (Figure S1). So obtained values were verified by DSC measurement of a model organogel ( $40 \mathrm{~g} \mathrm{~L}^{-1} \mathbf{C}_{\mathbf{1 2}}$-Cyc in cyclohexane). Herein, the temperature at which the gel started to break was defined as $T_{\text {gel }}$. Gel stability was controlled by taking the vial out of the heating

40 block and turning it upside down to find gravitational flow. Each measurement was made at least by duplicate and the average value reported. $T_{\text {gel }}$ values were found almost unaltered within a difference of $1-2^{\circ} \mathrm{C}$ after several heating-cooling cycles.

Oscillatory rheology was performed with an AR 2000 ${ }_{45}$ Advanced rheometer (TA Instruments) equipped with a Julabo C cooling system. A $1000 \mu \mathrm{m}$ gap setting and a torque of $5 \times 10^{-4}$ $\mathrm{N} / \mathrm{m}$ at $25^{\circ} \mathrm{C}$ were used for the measurements in a plain-plate $(40$ $\mathrm{mm}$, stainless steel). The following experiments were carried out for each sample, using ca. $2 \mathrm{~mL}$ total gel volume: a) Dynamic ${ }_{50}$ Strain Sweep (DSS): variation of $G^{\prime}$ (storage modulus) and $G^{\prime \prime}$ (loss modulus) with strain (from 0.01 to $100 \%$ ); b) Dynamic Frequency Sweep (DFS): variation of $G^{\prime}$ and $G^{\prime \prime}$ with frequency (from 0.1 to $10 \mathrm{~Hz}$ at $0.1 \%$ strain); c) Dynamic Time Sweep (DTS): variation of $G^{\prime}$ and $G^{\prime \prime}$ with time keeping the strain and 55 frequency values constant and within the linear viscoelastic regime as determined by DSS and DFS measurements (strain = $0.1 \%$ strain; frequency $=1 \mathrm{~Hz}$ ).

Fourier transform infrared (FT-IR) spectra were recorded at room temperature using an Excalibur FTS 3000 FT-IR ${ }_{60}$ spectrometer (Biorad) equipped with a single reflection ATR (attenuated total reflection) accessory (Golden Gate, Diamond).

X-Ray Diffraction spectroscopy (XRD) was performed on a STOE STADI P powder diffractometer (StartFragment Transmission mode, flat samples, Dectris MYTHEN $1 \mathrm{k}$ ${ }_{65}$ microstrip solid-state detector) with $\mathrm{CuK} \alpha$ radiation operated at $40 \mathrm{kV}$ and $40 \mathrm{~mA}$. Conditions were $0.015^{\circ}$, time $600 \mathrm{~s}$ per step, 2 theta range $0-90^{\circ}$.

Field Emission Scanning Electron Microscopy (FESEM, resolution $0.8 \mathrm{~nm}$ ) images of xerogels were obtained with a Carl 70 Zeiss Merlin, Field Emission Scanning Electron Microscope equipped with a digital camera and operated at an accelerating voltage of $10 \mathrm{kV}$. For visualization, samples were prepared by the freeze-drying (FD) method: An Eppendorf tube containing the corresponding gel-material $(100-200 \mu \mathrm{L})$ was frozen in liquid 75 nitrogen or dry ice/acetone and the solvent was immediately evaporated under reduced pressure $(0.6 \mathrm{mmHg})$ for 2 days at RT. The so obtained fibrous solid was placed on top of a tin plate and shielded by Pt ( $40 \mathrm{~mA}$ during $30 \mathrm{~s}$; film thickness $\approx 5 \mathrm{~nm}$ )

UV-Vis spectra of nanoparticle-containing gels were recorded 80 using an Ocean Optics Flame Miniature Spectrometer equipped with an Ocean Optics DH-2000-BAL light source and an Ocean Optics $1 \mathrm{~cm}$ cuvette holder. For measurements using $1 \mathrm{~mm}$ quartz glass cuvettes, nanoparticle-containing gels were diluted $1: 1(\mathrm{v} / \mathrm{v})$ or more when noted to minimize light scattering.

\section{${ }_{85}$ General procedure for the preparation of metallogels}

A weighed amount of the solid gelator $\left(\mathbf{C}_{\mathbf{1 2}^{-}}-\mathbf{C y c}\right.$ or click- $\mathbf{C}_{\mathbf{1 2}^{-}}$ Cyc) was placed into a in a $2 \mathrm{~mL}$ screw-capped vial $(4 \mathrm{~cm}$ length and $1 \mathrm{~cm}$ diameter). Then, the corresponding metal salt was added in its crystalline form. After the addition of the solvent, the 90 vials were closed with the cap and gently heated with a heat-gun until the solid material was completely dissolved. Cooling the mixtures to RT induced the formation of the metallogels. No control over temperature rate during the heating-cooling process was applied. The material was preliminarily classified as "gel" if 95 it did not exhibit gravitational flow upon turning the vial upsidedown at RT. The state was further confirmed by rheological measurements. Gelation time was checked by repeatedly moving the gel-containing vial until no gravitational flow was observed anymore.

\section{General procedure for in situ formation of Au nanoparticle-} containing gels

$\mathbf{C}_{12}-\mathbf{C y c}$, click- $\mathbf{C}_{12}-\mathbf{C y c}$ or a mixture of both compounds was weighed in a screw-capped vial (4 cm length and $1 \mathrm{~cm}$ diameter). After the subsequent addition of solid $\mathrm{HAuCl}_{4} \cdot 3 \mathrm{H}_{2} \mathrm{O}$, the 
corresponding solvent was added using a microliter syringe. Thereafter, $\mathrm{Et}_{3} \mathrm{~N}$ was added in one portion immediately leading to a color change, which was enhanced as the suspensions were heated and cooled down to RT affording opaque gels with dark 5 red-brown colors as described in the main text.

Photochemical reactions were performed under UV light $(\lambda=$ $245 \mathrm{~nm}$; light power $=8 \mathrm{~W}$ ) using a Rayonet RMR-600 Photochemical reactor at $35^{\circ} \mathrm{C}$ (chamber temperature).

\section{Results and discussion}

10 In a recent study, we have reported a comparative study of the gelation ability of the isosteres $\mathbf{C}_{\mathbf{1 2}}-\mathbf{C y c}$ and click-C $\mathbf{C}_{\mathbf{1 2}}-\mathbf{C y c}{ }^{33}$ Briefly, both gelators are able to form rapidly opaque and thermo-reversible organogels in various non-polar (e.g. cyclohexane, hexane, oils) and polar-protic (e.g. ethylene glycol, 15 methanol, nitromethane) solvents after a heating-cooling cycle. Furthermore, theoretical simulations revealed that click-C $\mathbf{C}_{\mathbf{1 2}}-\mathbf{C y c}$ can adopt a wide variety of interacting patterns, whose relative stability depend on the polarity of the environment, being in agreement with the experimental gelation data. ${ }^{33}$

20 Other important features of click- $\mathbf{C}_{\mathbf{1 2}}-\mathbf{C y c}$ for potential applications are its non-cytotoxic character and its phaseselective gelation of water-oil mixtures. In addition, triazole ring offers the versatility to bridge several metal centers and obtain unique polynuclear compounds. Thus, in this work we have

25 investigated and compared the formation of metallogels with both isosteres $\left(\mathbf{C}_{\mathbf{1 2}}\right.$-Cyc and click- $\left.\mathbf{C}_{\mathbf{1 2}}-\mathbf{C y c}\right)$, as well as the in situ formation and stabilization of gold nanoparticles embedded in the gel-phase network.

\section{Formation of metallogels}

30 The formation of metallogels using different transition metals and LMW ligands has a long history in supramolecular chemistry. ${ }^{39,40,41,42,43,44}$ Many of these metallogels have been used as matrices for the in situ formation of metal nanoparticles, mainly silver ${ }^{45,46,47,48,49}$ and gold 35 nanoparticles. ${ }^{50,51,52,53,54,55,56,57,58,59}$

In this study, both $\mathbf{C}_{\mathbf{1 2}}$-Cyc and click- $\mathbf{C}_{\mathbf{1 2}}$-Cyc yielded precipitates in the absence of metal ions in polar aprotic solvents such DMSO. However, stable opaque metallogels were obtained in these solvents via a heating-cooling cycle upon incorporation 40 of some metals. Materials that did not show gravitational flow upon turning the vial upside-down were initially classified as stable gels. The viscoelastic nature of some selected gels was later confirmed by oscillatory rheological experiments (vide infra). Specifically, $\mathbf{C}_{\mathbf{1 2}}$-Cyc enabled the formation of metallogels 45 with $\mathrm{Pd}^{\mathrm{II}}$ and $\mathrm{Au}^{\mathrm{III}}$ in DMSO and DMF, whereas click-C $\mathbf{C}_{\mathbf{1 2}}-\mathbf{C y c}$ formed metallogels in these solvents only in the presence of $\mathrm{Au}^{\text {III }}$ (Figure 2). The exact reason for such different behavior remains unclear at the moment. Experiments incorporating $\mathrm{Cu}^{\mathrm{II}}, \mathrm{Co}^{\mathrm{II}}$ or $\mathrm{Ni}^{\mathrm{II}}$ yielded viscous solutions instead of stable gels. At this point, 50 we selected DMF and $\mathrm{Au}^{\mathrm{III}}$ as model solvent and metal ion to perform additional studies.

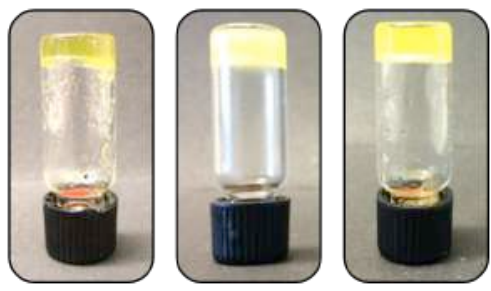

Fig. 2 Pictures of metallogels prepared from $\mathbf{C}_{12}-\mathbf{C y c}$ and click- $\mathbf{C}_{12}$-Cyc. 55 Left: $\mathbf{C}_{12}$-Cyc in DMSO $\left(c=24 \mathrm{mmol} \mathrm{L}{ }^{-1}\right), 12 \mathrm{~mol} \% \mathrm{PdCl}_{2}$. Middle: $\mathbf{C}_{12}-$ Cyc in DMF $\left(c=41 \mathrm{mmol} \mathrm{L}^{-1}\right), 90 \mathrm{~mol} \% \mathrm{HAuCl}_{4} \cdot 3 \mathrm{H}_{2} \mathrm{O}$. Right: click$\mathbf{C}_{12}$-Cyc in DMF $\left(c=43 \mathrm{mmol} \mathrm{L}^{-1}\right), 89 \mathrm{~mol} \%$.

In order to optimize the gelator:metal molar ratio we 60 investigated the evolution of both $T_{\text {gel }}$ and gelation time values with gelator and metal salt concentrations for both $\mathbf{C}_{\mathbf{1 2}}$-Cyc and click- $\mathbf{C}_{\mathbf{1 2}}$-Cyc in DMF (Figure S2, ESI). In all cases, the gelator concentration was found to have a much higher influence on the gel stability than the concentration of $\mathrm{HAuCl}_{4} \cdot 3 \mathrm{H}_{2} \mathrm{O}$. At high ${ }_{65}$ concentrations $\left(>40 \mathrm{mmol} \mathrm{L}^{-1}\right.$ ) of $\mathbf{C}_{\mathbf{1 2}}$-Cyc, both $T_{\text {gel }}$ and gelation kinetics increased with increasing gold concentrations whereas this influence was less pronounced at lower gelator concentrations (Figure S2, ESI). Regarding click- $\mathbf{C}_{\mathbf{1 2}}-\mathbf{C y c}, T_{\text {gel }}$ increased with the metal concentration at high gelator 70 concentrations, while it decreased at low gelator concentrations (Figure S2, ESI). However, the gelation time was only influenced by the metal concentration at very high amounts of gelator (Figure S2, ESI). Concerning a nearly optimal gelator concentration of $40 \mathrm{mmol} \mathrm{L}^{-1}$, all trends could be considered 75 linear and flat leading to a free variability of the gold concentration without decreasing the gel stability. Comparing the gels made of $\mathbf{C}_{\mathbf{1 2}}$-Cyc vs click- $\mathbf{C}_{\mathbf{1 2}}$-Cyc in the presence of $\mathrm{HAuCl}_{4} \cdot 3 \mathrm{H}_{2} \mathrm{O}$, the former showed higher $T_{\text {gel }}$ values $\left(62{ }^{\circ} \mathrm{C}\right.$ compared to $41{ }^{\circ} \mathrm{C}$ in presence of $60 \mathrm{mmol} \mathrm{L}^{-1}$ gelator and $\left.{ }_{80} 40 \mathrm{mmol} \mathrm{L}^{-1} \mathrm{Au}\right)$ and 5-9 times shorter gelation times $(110 \mathrm{~s}$ compared to $950 \mathrm{~s}$ in presence of $30 \mathrm{mmol} \mathrm{L}^{-1}$ gelator and $40 \mathrm{mmol} \mathrm{L}^{-1} \mathrm{Au}$ ). Table 1 outlines the CGC, $T_{\text {gel }}$ and gelation time values for the gels made of $\mathbf{C}_{\mathbf{1 2}}-\mathbf{C y c}$ and click- $\mathbf{C}_{\mathbf{1 2}}$-Cyc with a 1:1 molar ratio of gelator and gold. It is worth mentioning that 85 the CGC of $\mathbf{C}_{12}$-Cyc in DMF in the presence of $\mathrm{HAuCl}_{4} \cdot 3 \mathrm{H}_{2} \mathrm{O}$ was ca. $40 \%$ lower than in the absence of the metal salt (i.e. 12 $\mathrm{mM}=6 \mathrm{~g} \mathrm{~L}^{-1}$ vs $10 \mathrm{~g} \mathrm{~L}^{-1}$, respectively).

Table 1 CGC, $T_{\text {gel }}$ and gelation time values of metallogels made of $\mathbf{C}_{12}-$ ${ }_{90} \mathbf{C y c}$ and click- $\mathrm{C}_{12}-\mathrm{Cyc}$ with $\mathrm{HAuCl}_{4} \cdot 3 \mathrm{H}_{2} \mathrm{O}(100 \mathrm{~mol} \%)$. Molar ratio gelator: $\mathrm{Au}=1: 1$.

\begin{tabular}{cccc}
\hline Gelator & \multirow{2}{*}{$\mathrm{CGC}\left(\mathrm{mmol} \mathrm{L}^{-1}\right)$} & Gelation time $(\min )$ & $T_{\text {gel }}\left({ }^{\circ} \mathrm{C}\right)$ \\
\hline $\mathbf{C}_{\mathbf{1 2}}$-Cyc & $12 \pm 2$ & $2.5 \pm 0.5$ & $37 \pm 2$ \\
\hline click-C $\mathbf{C}_{\mathbf{1 2}}$-Cyc & $29 \pm 2$ & $13 \pm 3$ & $32 \pm 1$ \\
\hline
\end{tabular}

FT-IR and XRD spectroscopic studies of the metallo-xerogels revealed similar aggregation patterns and the presence of 95 crystalline domains as observed with the metal-free organogels (Figures S3-S4, ESI). As shown in Figure 3, these metallogels also responded to a variety of external stimuli.

The metallogels prepared from both isosteres showed full thermal reversibility and remained stable over at least one month. 100 The addition of $\mathrm{NaOH}(1 \mathrm{M})$ led to partial collapse of the 
yellowish gels, which became blackish presumably due to the reduction of $\mathrm{Au}^{\mathrm{III}}$ to $\mathrm{Au}^{0}$ (Figure 3). Mechanical agitation caused partial dissolution only of the metallogel made of click- $\mathbf{C}_{\mathbf{1 2}}-\mathbf{C y c}$. Moreover, the addition of concentrated acetic acid, water, PBS $5(0.1 \mathrm{M}, \mathrm{pH} 7.0)$ and $\mathrm{HCl}(1 \mathrm{M})$ led to partial dissolution of both gels, albeit the process was faster in the case of click- $\mathbf{C}_{\mathbf{1 2}}$-Cyc. This observation differed from the metal-free experiments, where only acetic acid among these additives showed an impact on the gel stability. The consistence of the gels remained upon addition 10 of solid additives such as $\mathrm{FeCl}_{3}, \mathrm{PdCl}_{2}$ and $\mathrm{CuSO}_{4} \cdot 5 \mathrm{H}_{2} \mathrm{O}$. These salts slowly diffused through the gel network, which could be visually monitored by color change of the bulk gels (Figure 3). The gels remained stable in consistence and visual appearance under sonication and after the addition of other additives such as ${ }_{15} \mathrm{NaCl}$ and $\mathrm{MeOH}$. Interestingly, UV irradiation $(\lambda=254 \mathrm{~nm})$ over a period of $6 \mathrm{~h}$ caused initial discoloration of the gels. After 30 min the gel made of click- $\mathbf{C}_{\mathbf{1 2}}$-Cyc became colorless and was thereafter found to develop a blackish brown appearance with increasing intensity over time (Figure S5, ESI). The same 20 behavior was found for the gel made of $\mathbf{C}_{\mathbf{1 2}}$-Cyc, albeit the process was much slower leading to full discoloration after $120 \mathrm{~min}$ and a final dark yellow color after $6 \mathrm{~h}$. These observations were in agreement with the generation of gold nanoparticles of different sizes as confirmed later by plasmonic ${ }_{25}$ resonances (vide infra).$^{60}$ These results suggest that both isosteres display intrinsic and significant reduction potentials.

A)

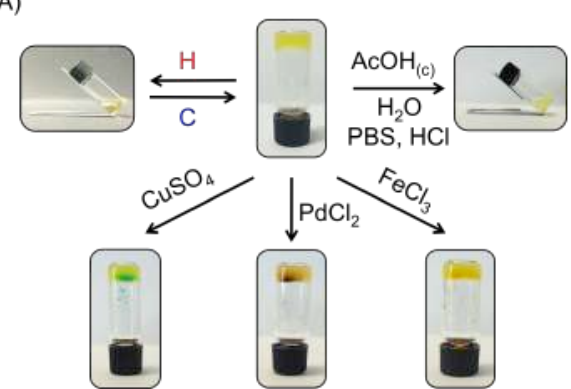

B)

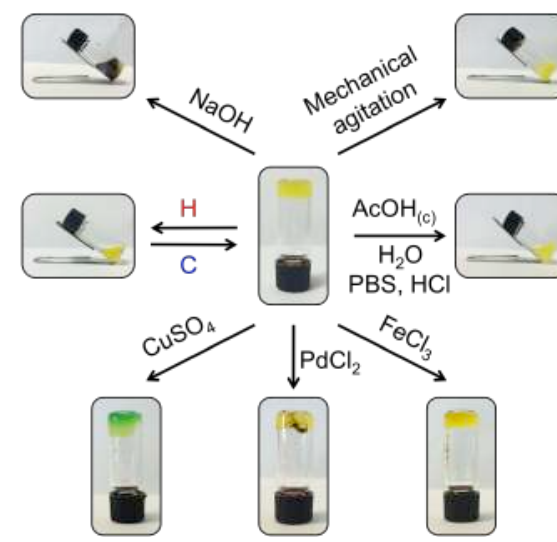

Fig. 3 Stimuli responsive map of metallogels made of (A) $\mathbf{C}_{12}$-Cyc and 30 (B) click- $\mathbf{C}_{12}$-Cyc (bottom) in the presence of $\mathrm{HAuCl}_{4} \cdot 3 \mathrm{H}_{2} \mathrm{O}$ in DMF (gelator:metal $=1: 1$ molar ratio; $c=40 \mathrm{~g} \mathrm{~L}^{-1}$ ). Abbreviation: $\mathrm{H}=$ heating; $\mathrm{C}=$ cooling. $[\mathrm{PBS}]=0.1 \mathrm{M} ;[\mathrm{HCl}]=1 \mathrm{M} ;[\mathrm{NaOH}]=1$ M. Note: Partial gel with a blackish appearance was also observed in the case of the metallogel made of $\mathbf{C}_{12}-\mathbf{C y c}$ upon 35 treatment with $\mathrm{NaOH}$ (picture not shown).

In terms of flow properties, dynamic oscillatory rheological experiments (i.e. DFS, DSS, DTS) confirmed the viscoelastic nature of selected metallogels and revealed a storage modulus $G^{\prime}$ 40 higher than the loss modulus $G^{\prime \prime}$. The difference between $G^{\prime}$ and $G^{\prime \prime}$ is much higher in the case of $\mathbf{C}_{\mathbf{1 2}}$-Cyc metallogel. Overall, the results showed a higher mechanical strength of the $\mathbf{C}_{12}$-Cyc metallogel compared to the click- $\mathbf{C}_{\mathbf{1 2}}$-Cyc metallogel, as indicated by higher absolute $G$ ' and lower $\tan \delta$ values (Table 2; ${ }_{45}$ Figure S6, ESI). However, the yield stress $(\gamma)$ was higher for the gel made of click- $\mathbf{C}_{12}$-Cyc (i.e. $2.2 \%$ vs $1.8 \%$ ). The same trends were in agreement with those observed in the metal-free experiments. The most important effect of the incorporation of the metal into the gels was observed in the damping properties of 50 the material, making the metallogel made of click- $\mathbf{C}_{\mathbf{1 2}}$-Cyc less elastic compared to its metal-free version. The opposite behavior was observed in the case of $\mathbf{C}_{\mathbf{1 2}}$-Cyc, where the potential to store the load increased upon incorporation of the metal.

55 Table 2 Rheological data of metallogels made of $\mathbf{C}_{12}$ - $\mathbf{C y c}$ and click- $\mathbf{C}_{12-}$ Cyc $\left(c=40 \mathrm{~g} \mathrm{~L}^{-1}\right)$ in DMF incorporating $\mathrm{HAuCl}_{4} \cdot 3 \mathrm{H}_{2} \mathrm{O}\left(c=40 \mathrm{~g} \mathrm{~L}^{-1}\right)$.

\begin{tabular}{|c|c|c|c|c|}
\hline Gelator & $G^{\prime}(\mathrm{kPa})$ & $G^{\prime \prime}(\mathrm{kPa})$ & $\tan \delta$ & $\gamma(\%)$ \\
\hline $\mathrm{C}_{12}$-Cyc & 12 & 1.4 & 0.12 & 1.8 \\
\hline click- $\mathrm{C}_{12}-\mathrm{Cyc}$ & 3.5 & 1.4 & 0.4 & 2.2 \\
\hline
\end{tabular}

\section{Formation of gold nanoparticles-containing gels}

Other metallogels have been already used for catalytic purposes ${ }^{12}$ as well as for the formation and stabilization of metal 60 nanoparticles (NPs). ${ }^{61}$ Within this context, we decided to check also the possibility to generate gold NPs by intragel reduction of $\mathrm{HAuCl}_{4} \cdot 3 \mathrm{H}_{2} \mathrm{O}$. First of all, we investigated the solubility properties of the metal salt in various solvents and the stability of bulk gels in the presence of the metal salt (Table S1-S2, ESI).

${ }_{65}$ From these preliminary experiments, propan-2-ol, acetone, NMP and DMF yielded stable opaque gels, suggesting their potential use as solvent for metal reduction. A brown appearance of the gel was observed directly in the case of propan-2-ol presumably due to its intrinsic reductive character. ${ }^{62}$

70 Although UV irradiation was found to induce metal reduction, the method was slow and less reproducible than using external reducing agents such as $\mathrm{NaBH}_{4}, \mathrm{Et}_{3} \mathrm{~N}$ or hydroquinone (Table $\mathrm{S} 3$, ESI). These reducing agents showed solubility in DMF both at $\mathrm{RT}$ and $80^{\circ} \mathrm{C}$, in contrast to other common reducing agents such 75 as sodium citrate or sodium ascorbate. The metal reduction was investigated using a fixed gelator concentration $\left(c=40 \mathrm{mmol} \mathrm{L}^{-}\right.$ ${ }^{1}$ ), which previously demonstrated to support a consistent gel stability under varying concentrations of $\mathrm{HAuCl}_{4} \cdot 3 \mathrm{H}_{2} \mathrm{O}$. Thus, the concentration of $\mathrm{HAuCl}_{4} \cdot 3 \mathrm{H}_{2} \mathrm{O}$ was varied between 20 and ${ }_{80} 60 \mathrm{mmol} \mathrm{L}^{-1}$ during the experiments. Before the application of a heating-cooling cycle, $\mathrm{Et}_{3} \mathrm{~N}$ was added to the suspension of gelator and $\mathrm{HAuCl}_{4} \cdot 3 \mathrm{H}_{2} \mathrm{O}\left(\right.$ i.e. $\left.\left[\mathrm{Et}_{3} \mathrm{~N}\right]=10 \times[\mathrm{Au}]\right)$. Immediately thereafter, a reddish color was developed in all samples containing the metal salt. Subsequent application of a heating${ }_{85}$ cooling cycle yielded the formation of stable dark reddish-brown gels as expected due to the formation of the corresponding gold 
NPs (Figure 4). The intensity of the dark color increased gradually with the concentration of metal salt. Both $\mathbf{C}_{12}-\mathbf{C y c}$ and click- $\mathbf{C}_{\mathbf{1 2}}$-Cyc were tested individually and also as a 50:50 mixture (molar ratio) in order to study the influence of the 5 surrounding gelator network on the resulting nanoparticles. In general, both gelation kinetics and gel strengths were visually enhanced by the presence of gold in the formulations. Importantly, all gels remained stable after the reduction process was complete; indicating the robustness of the formulations for 10 the in situ formation of NPs embedded in the viscoelastic networks. It is worth mentioning that other metallogels have also been used for the synthesis of NPs in both apolar organic solvents $^{51}$ and in water. ${ }^{54,57,58}$

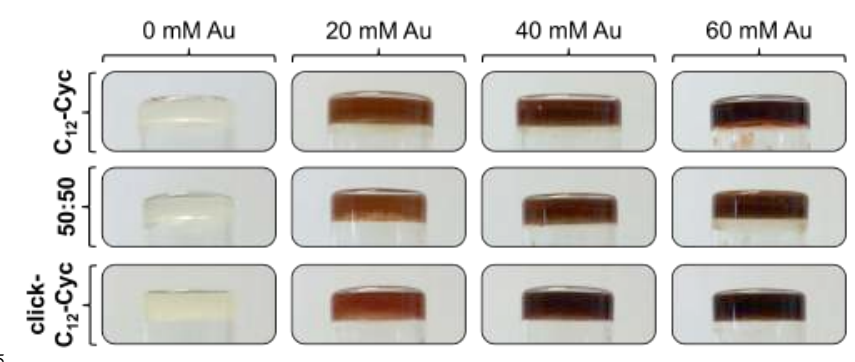

Fig. 4 Formation of gold nanoparticles embedded in stable gel matrices made of $\mathbf{C}_{\mathbf{1 2}}$-Cyc, click- $\mathbf{C}_{\mathbf{1 2}}-\mathbf{C y c}$ and an equimolar mixture of both gelators $\left(c=40 \mathrm{~g} \mathrm{~L}^{-1}\right)$ with different concentrations of $\mathrm{HAuCl}_{4} \cdot 3 \mathrm{H}_{2} \mathrm{O}$ and $\mathrm{Et}_{3} \mathrm{~N}$ (10 equiv with respect to metal salt). Samples in the absence of 20 metal salt were also treated with $\mathrm{Et}_{3} \mathrm{~N}\left(c=400 \mathrm{~g} \mathrm{~L}^{-1}\right)$ as control. The mixture 50:50 corresponds to an equimolar mixture of both gelators (50 $\mathrm{mol} \%$ each).

Finally, in order to gain information about the size distribution 25 of the formed nanoparticles, we carried out UV-vis studies of the NPs-containing gels and electron microscopy imaging of the corresponding xerogels. The plasmon resonance band centered at ca. $520 \mathrm{~nm}$ was observed in the UV-vis spectra of all metallogels made of pure isosteres or an equimolar mixture of click- $\mathbf{C}_{\mathbf{1 2}}-\mathbf{C y c}$ 30 and $\mathbf{C}_{\mathbf{1 2}}$-Cyc, suggesting the formation of NPs with similar size distribution regardless the gold concentration and the nature of the gelator system. The plasmon resonance was particularly pronounced in the samples with $[\mathrm{Au}]=60 \mathrm{mmol} \mathrm{L}^{-1}$ (Figure 5; Figure S7, ESI). These results are in good agreement with 35 previous studies reported in the literature. ${ }^{63}$

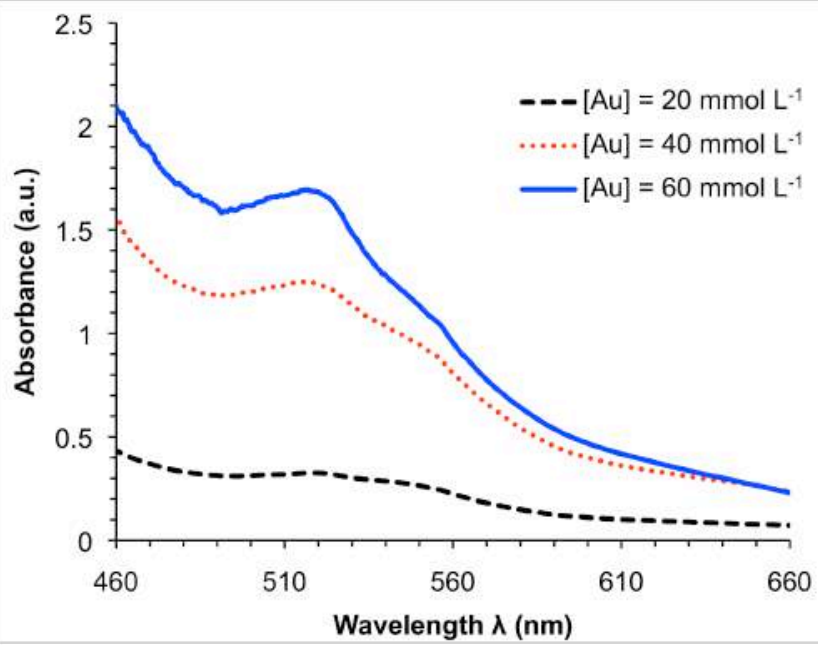

Fig. 5 UV-vis spectra of Au NPs dispersed in the gel matrix made of click- $\mathbf{C}_{12}$-Cyc $\left(c=40 \mathrm{mmol} \mathrm{L}^{-1}\right)$. The NPs were obtained using different 40 concentrations of $\mathrm{HAuCl}_{4} \cdot 3 \mathrm{H}_{2} \mathrm{O}$ and $\mathrm{Et}_{3} \mathrm{~N}$ as described in Figure 4.

FESEM images of the xero-metallogel made of click- $\mathbf{C}_{\mathbf{1 2}}$-Cyc, $\mathrm{HAuCl}_{4} \cdot 3 \mathrm{H}_{2} \mathrm{O}$ and $\mathrm{Et}_{3} \mathrm{~N}$ after the reduction was complete showed a band-structure morphology (Figure 6A-D; Figure S8, ESI) that 45 reminded those previously observed for the metal-free xerogel prepared in nitromethane. The formed gold NPs dispersed through the gel network were clearly visualized by TEM imaging (Figure 6E-J; Figures S9-S11, ESI). Non-aggregated NPs were equally distributed over the ordered template networks regardless ${ }_{50}$ the gelator system, albeit they were polydisperse in nature. The gel made of an equimolar mixture of $\mathbf{C}_{\mathbf{1 2}}-\mathbf{C y c}$ and click-C $\mathbf{C}_{\mathbf{1 2}}-\mathbf{C y c}$ allowed for the formation of gold NPs with the smallest size distribution centered around $4 \mathrm{~nm}$ (Figure S12, ESI). Similar particle sizes with a resonance band centered at $520 \mathrm{~nm}$ have 55 been also reported with steroidal-based network. ${ }^{63}$ The average particle sizes of the three gelators systems were $4.7 \pm 2.3 \mathrm{~nm}$ $\left(\mathbf{C}_{\mathbf{1 2}}\right.$-Cyc), $4.0 \pm 1.7 \mathrm{~nm}\left(50: 50, \mathbf{C}_{\mathbf{1 2}}\right.$-Cyc:click-C $\left.\mathbf{1}_{\mathbf{1 2}}-\mathbf{C y c}\right)$, and 4.6 $\pm 2.3 \mathrm{~nm}$ (click- $\mathbf{C}_{\mathbf{1 2}}$-Cyc). In general, these NPs are ca. 1-2 times larger in diameter than those obtained with metallogels made of ${ }_{60}$ gelators bearing thiol ${ }^{50,51}$ or disulfide ${ }^{53}$ moieties. However, much larger $\mathrm{Au}$ NPs have been also fabricated using urea-based gelators ${ }^{52,56}$ and hydrogels. ${ }^{54}$ In addition, monodispersed Au NPs of ca. $5 \mathrm{~nm}$ with icosahedral geometry have been reported using bis-imidazolium amphiphile-based hydrogels. ${ }^{58}$ In many cases,

${ }_{65} \mathrm{NaBH}_{4}$ is used as reducing agent, while we used $\mathrm{Et}_{3} \mathrm{~N}$ that can be easily removed by evaporation during the preparation of the xerogels. Importantly, the gels containing non-agglomerated NPs could be restored by simple addition of DMF to the xerogel and applying a heating-cooling cycle. Further characterization of the 70 50:50 mixture is still necessary in order to understand the effect of the co-assembly process in the formation of the NPs. In addition, we confirmed the laser-triggered degelation of a gold NPs containing gel made of click- $\mathbf{C}_{\mathbf{1 2}}$-Cyc $\left(c=40 \mathrm{~g} \mathrm{~L}^{-1}\right)$ in DMF incorporating $\mathrm{HAuCl}_{4} \cdot 3 \mathrm{H}_{2} \mathrm{O}$ (molar ratio gelator: $\mathrm{Au}=1: 1$ ).

75 Finally, in agreement with previous studies, ${ }^{64}$ the bulk gel with a $T_{\text {gel }}$ of ca. $40^{\circ} \mathrm{C}$ collapsed after 35 min of green laser exposure ( $\lambda$ $=532 \mathrm{~nm}, 50 \mathrm{~mW} / \mathrm{cm}^{2}$ ) due to the photothermal properties of the $\mathrm{Au}$ NPs. As expected, the gel-phase was slowly reformed when 

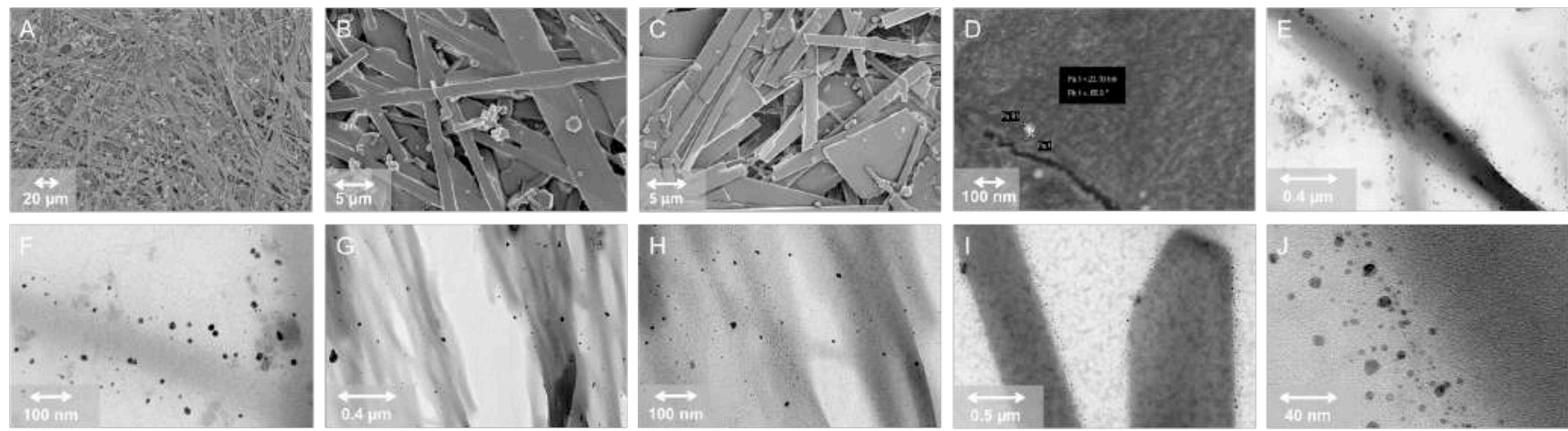

5 Fig. 6 A-D) Representative FESEM pictures of xerogels obtained by freeze-drying the corresponding metallogels made of click- $\mathbf{C}_{12}-\mathbf{C y c}^{-}(c=40 \mathrm{mmol} \mathrm{L}$

$\left.{ }^{1}\right), \mathrm{HAuCl}_{4} \cdot 3 \mathrm{H}_{2} \mathrm{O}\left(c=40 \mathrm{mmol} \mathrm{L}^{-1}\right)$, and $\mathrm{Et}_{3} \mathrm{~N}\left(c=400 \mathrm{mmol} \mathrm{L}^{-1}\right)$ in DMF. E-F) Representative TEM photographs of the gel prepared from $\mathbf{C}_{12}-\mathrm{Cyc}(c=$ $\left.40 \mathrm{mmol} \mathrm{L}^{-1}\right), \mathrm{HAuCl}_{4} \cdot 3 \mathrm{H}_{2} \mathrm{O}\left(c=40 \mathrm{mmol} \mathrm{L}^{-1}\right)$, and $\mathrm{Et}_{3} \mathrm{~N}\left(c=400 \mathrm{mmol} \mathrm{L}^{-1}\right)$ in DMF. G-H) Representative TEM photographs of the gel prepared from $\mathbf{C}_{12}$-Cyc $\left(c=20 \mathrm{mmol} \mathrm{L}^{-1}\right)$ and click-C $\mathbf{1 2}_{12}$-Cyc $\left(c=20 \mathrm{mmol} \mathrm{L}^{-1}\right), \mathrm{HAuCl}_{4} \cdot 3 \mathrm{H}_{2} \mathrm{O}\left(c=40 \mathrm{mmol} \mathrm{L}^{-1}\right)$, and $\mathrm{Et}_{3} \mathrm{~N}\left(c=400 \mathrm{mmol} \mathrm{L}^{-1}\right)$ in DMF. I-J Representative TEM photographs of the gel prepared from click- $\mathrm{C}_{12}-\mathrm{Cyc}\left(c=40 \mathrm{mmol} \mathrm{L}{ }^{-1}\right), \mathrm{HAuCl}_{4} \cdot 3 \mathrm{H}_{2} \mathrm{O}\left(c=40 \mathrm{mmol} \mathrm{L}^{-1}\right)$, and Et $\mathrm{Et}_{3}\left(c=400 \mathrm{mmol} \mathrm{L}^{-}\right.$ $\left.10^{1}\right)$ in DMF. See ESI for additional pictures. Note: Differences observed between SEM and TEM images with respect to the morphology of the matrix (i.e. microscale crystalline tapes $v s$ nanoscale fibers) could be due to modifications occurred during the preparation of the samples such as partial crystallization of the gelator.

\section{Conclusions}

In conclusion, the isosteric substitution of the two amide groups 15 presented in the LMW gelator $N, N^{\prime}-((1 S, 2 S)$-cyclohexane-1,2diyl)didodecanamide $\left(\mathbf{C}_{\mathbf{1 2}}\right.$-Cyc) by 1,2,3-triazole rings afforded the corresponding isosteric gelator $\left(\mathbf{c l i c k}-\mathbf{C}_{\mathbf{1 2}}-\mathbf{C y c}\right)$. This approach allows for fine-tuning the properties of the corresponding organo- and metallogels. The foregoing results 20 demonstrate that $\mathbf{C}_{\mathbf{1 2}}$-Cyc can form stable and reversible metallogels with $\mathrm{Pd}^{\mathrm{II}}$ and $\mathrm{Au}^{\mathrm{III}}$ in DMSO and DMF, whereas click- $\mathbf{C}_{\mathbf{1 2}}$-Cyc forms metallogels in these solvents only in the presence of $\mathrm{Au}^{\mathrm{III}}$. Comparing the gels made of $\mathbf{C}_{\mathbf{1 2}}$-Cyc $v s$ click$\mathbf{C}_{12}$-Cyc in the presence of $\mathrm{HAuCl}_{4} \cdot 3 \mathrm{H}_{2} \mathrm{O}$, the former showed ${ }_{25}$ higher $T_{\text {gel }}$ values and 5-8 times shorter gelation times. Furthermore, the incorporation of $\mathrm{Et}_{3} \mathrm{~N}$ as reducing agent in the formulations facilitated the in situ formation of gold NPs embedded in the viscoelastic networks. The plasmon resonance band centered at ca. $520 \mathrm{~nm}$ was observed in the UV-vis spectra 30 of all metallogels made of pure isosteres or an equimolar mixture of click- $\mathbf{C}_{\mathbf{1 2}}$-Cyc and $\mathbf{C}_{\mathbf{1 2}}$-Cyc, suggesting the formation of NPs with similar size distribution regardless the gold concentration and the nature of the gelator system. The formed gold NPs dispersed through the gel network were clearly visualized by

35 TEM imaging, showing non-aggregated NPs equally distributed over the ordered template networks. It is important to highlight that both the solvent (DMF) and the reducing agent $\left(\mathrm{Et}_{3} \mathrm{~N}\right)$ used in these experiments could be evaporated during the preparation of the xerogels by freeze-drying for $5 \mathrm{~h}$ under reduced pressure.

40 This process afforded a non-toxic and chemically inert network template in which Au NPs were equally distributed. Subsequent addition of DMF to the xerogels following by heating enabled again a dark-red isotropic solution without agglomeration of the NPs.

\section{${ }_{45}$ Acknowledgements}

This work was supported by the University of Regensburg, the
Deutsche Forschungsgemeinschaft (DFG), and Fondecyt iniciación 11160707. D.D.D. thanks the DFG for the Heisenberg Professorship Award.

\section{${ }_{50}$ Notes and references}

1 J. H. van Esch and B. L. Feringa, Angew. Chem., Int. Ed., 2000, 39, 2263-2266.

2 M. George and R. G. Weiss, Acc. Chem. Res., 2006, 39, 489-497.

3 S. Banerjee, R. K. Das and U. Maitra, J. Mater. Chem., 2009, 19, 6649-6687.

4 R. V. Ulijn and A. M. Smith, Chem. Soc. Rev., 2008, 37, 664-675.

5 M. O. M. Piepenbrock, G. O. Lloyd, N. Clarke and J. W. Steed, Chem. Rev., 2010, 110, 1960-2004.

6 S. Li, V. T. John, G. C. Irvin, S. H. Bachakonda, G. L. McPherson and C. J. O'Connor, J. Appl. Phys., 1999, 85, 5965-5967.

7 T. Kato, Science, 2002, 295, 2414-2418.

8 J. Puigmarti-Luis, V. Laukhin, A. P. del Pino, J. Vidal-Gancedo, C. Rovira, E. Laukhina and D. B. Amabilino, Angew. Chem., Int. Ed., 2007, 46, 238-241.

9 W. Kubo, S. Kambe, S. Nakade, T. Kitamura, K. Hanabusa, Y. Wada and S. Yanagida, J. Phys. Chem. B, 2003, 107, 4374-4381.

10 N. A. Peppas, P. Bures, W. Leobandung, H. Ichikawa, Eur. J. Pharm. Biopharm., 2000, 50, 27-46.

11 A. Friggeri, K. J. C. van Bommel and S. Shinkai, in Molecular Gels: Materials with Self-Assembled Fibrillar Networks; R. G. Weiss and P. Terech, (Eds.); Springer: Dordrecht, 2006; pp 857-893.

12 D. D. Díaz, D. Kühbeck and R. J. Koopmans, Chem. Soc. Rev., 2011, 40, 427-448.

13 E. M. Schön, E. Marqués-López, R. P. Herrera, C. Alemán and D. D. Díaz, Chem. Eur. J., 2014, 20, 10720-10731.

14 T. Patrick, Semin. Cutan. Med. Surg., 2004, 23, 233-235.

15 D. J. Abdallah and R. G. Weiss, Adv. Mater., 2000, 12, 1237-1247.

16 S.-k. Ahn, R. M. Kasi, S.-C. Kim, N. Sharma and Y. Zhou, Soft Matter, 2008, 4, 1151-1157.

17 Vögtle, F. Supramolecular Chemistry, Wiley, Chichester, 1991.

18 Lehn, J.-M. Supramolecular Chemistry: Concepts and Perspectives; VCH Weinheim, 1995.

19 R. G. Weiss and P. Terech, P. Molecular Gels: Materials with SelfAssembled Fibrillar Networks; Springer, New York, 2006.

20 J. van Esch and S. D. Feyter, Chem. Eur. J., 2006, 3, 1238-1243. 
21 K. Yoza, N. Amanokura, Y. Ono, T. Akao, H. Shinmori, M. Takeuchi, S. Shinkai and D. N. Reinhoudt, Chem. Eur. J., 1999, 5, 2722-2729.

22 J. Chen and A. J. McNeil, J. Am. Chem. Soc., 2008, 130, 1649616497.

23 M. H. Filby and J. W. Steed, Coord. Chem. Rev., 2006, 250, 32003218.

24 F. Fages, Angew. Chem. Int. Ed., 2006, 45, 1680-1682.

25 A. Y.-Y. Tam and V. W.-W. Yam, Chem. Soc. Rev., 2013, 42, 15401567.

26 J. Zhang and C. Y. Su, Coord. Chem. Rev., 2013, 257, 1373-1408.

27 M. Häring and D. D. Díaz, Chem. Commun., 2016, 52, 13068-13081.

28 I. Langmuir, J. Am. Chem. Soc., 1919, 41, 1543-1559.

29 J. Bachl, J. Mayr, F. J. Sayago, C. Cativiela and D. D. Díaz, Chem. Commun., 2015, 51, 5294-5297.

30 A. Burger, Prog Drug Res., 1991, 37, 287-371.

31 Bioisosteres in Medicinal Chemistry, ed. N. Brown,Wiley-VCH, Weinheim, 2012, vol. 54.

32 M. Häring, S. K. Nandi, J. Rodríguez-López, D. Haldar, V. S. Martín, A. D. Lozano-Gorrín, C. Saldías and D. D. Díaz, ACS Omega, 2019, 4, 2111-2117.

33 M. Tautz, J. Torras, S. Grijalvo, R. Eritja, C. Saldías, C. Alemán and D. D. Díaz, RSC Adv., 2019, DOI: 10.1039/c9ra03316e.

34 K. Hanabusa, M. Yamada, M. Kimura and H. Shirai, Angew. Chem. Int. Ed., 1996, 35, 1949-1951.

35 N. Zweep, A. Hopkinson, A. Meetsma, W. R. Browne, B. L. Feringa and J. H. van Esch, Langmuir, 2009, 25, 8802-8809.

36 H. Sato, T. Nakae, K. Morimoto and K. Tamura, Org. Biomol. Chem., 2012, 10, 1581-1586.

37 B. T. Kato, T. Kutsuna, K. Hanabusa and M. Ukon, Liq. Cryst., 1998, 606-608.

38 S. Yajima, K. Takami, R. Ooue and K. Kimura, Analyst, 2011, 136, 5131-5133.

39 J. Yuan, X. Fang, L. Zhang, G. Hong, Y. Lin, Q. Zheng, Y. Xu, Y. Ruan, W. Weng, H. Xia and G. Chen, J. Mater. Chem., 2012, 22, $11515-11522$

40 W. Weng, J. B. Beck, A. M. Jamieson and S. J. Rowan, J. Am. Chem. Soc., 2006, 128, 11663-11672.

41 S. Saha, J. Bachl, T. Kundu, D. D. Díaz and R. Banerjee, Chem. Commun., 2014, 50, 3004-3006.

42 S. Saha, E. M. Schön, C. Cativiela, D. D. Díaz and R. Banerjee, Chem. Eur. J., 2013, 19, 9562-9568.

43 A. R. Hirst, B. Escuder, J. F. Miravet and D. K. Smith, Angew. Chem. Int. Ed., 2008, 47, 8002-8018.

44 S. Bhattacharya and S. K. Samanta, Chem. Rev., 2016, 116, 1196712028

45 S. Dutta, A. Shome, T. Kar and P. K. Das, Langmuir, 2011, 27, 5000-5008.

46 R. Wang, J. Cui, X. Wan and J. Zhang, Chem. Commun., 2019, 55, 4949-4952.

47 M. Paul, K. Sarkar and P. Dastidar, Chem. Eur. J., 2015, 21, 255268.

48 P. Biswas, S. Ganguly and P. Dastidar, Chem. Asian J., 2018, 13, 1941-1949.

49 P. Slavík, D. W. Kurba and D. K. Smith, Chem. Sci., 2018, 9, 86738681 .

50 M. Kimura, S. Kobayashi, T. Kuroda, K. Hanabusa and H. Shirai, Adv. Mater., 2004, 16, 335-338.

51 L.-s. Li and S. I. Stupp, Angew. Chem. Int. Ed., 2005, 44, 1833-1836.

52 P. K. Vemula and G. John, Chem. Commun., 2006, 2218-2220.

53 J. van Herrikhuyzen, S. J. George, M. R. J. Vos, N. A. J. M. Sommerdijk, A. Ajayaghosh, S. C. J. Meskers and A. P. H. J. Schenning, Angew. Chem. Int. Ed., 2007, 46, 1825-1828.

54 R. N. Mitra and P. K. Das, J. Phys. Chem. C, 2008, 112, 8159-8166.

55 V. R. R. Kumar, V. Sajini, T. S. Sreeprasad, V. K. Praveen, A. Ajayaghosh and T. Pradeep, Chem. Asian J., 2009, 4, 840-848.

56 I. A. Coates and D. K. Smith, J. Mater. Chem., 2010, 20, 6696-6702.
57 J.-S. Shen, Y.-L. Chen, J.-L. Huang, J.-D. Chen, C. Zhao, Y.-Q. Zheng, T. Yu, Y. Yang and H.-W. Zhang, Soft Matter, 2013, 9, 2017-2023.

58 M. Rodrigues, A. Genç, J. Arbiol, D. B. Amabilino and L. PérezGarcía, J. Coll. Interface Sci., 2015, 446, 53-58.

59 B. O. Okesola, S. K. Suravaram, A. Parkin and D. K. Smith, Angew. Chem. Int. Ed., 2016, 55, 183-187.

60 K. Mallick, Z. L. Wang and T. Pal, J. Photochem. Photobiol. A Chem., 2001, 140, 75-80.

61 P. K. Vemula, U. Aslam, V. A. Mallia and G. John, Chem. Mater., 2007, 19, 138-140.

62 E. J. Campbell, H. Zhou and S. T. Nguyen, Org. Lett., 2001, 3, 23912393.

63 S. Bhat, U. Maitra and R. V. April, Chem. Mater., 2006, 18, 42244226.

64 H. Erdogan, H. Sakalak, M. S. Yavuz and G. Demirel, Langmuir, 2013, 29, 6975-6982. 